\title{
A case of hip joint septic arthritis as a complication of a prostate abscess
}

\author{
Themistoklis Vampertzis', Eirini losifidou ${ }^{1}$, Christina Barmpagianni², Dimitrios Takis', \\ Nikolaos Manidakis', Georgios Zervopoulos ${ }^{3}$, Stergios Papastergiou ${ }^{1}$
}

'Department of Orthopedics, Unit for Sports Medicine, Agios Pavlos G.H.Th., Thessaloniki, Greece; ${ }^{2}$ Trauma and Orthopaedics, Northwick Park Hospital, London North West University Health Care NHS Trust, UK; ${ }^{3}$ Department of Urology, Agios Pavlos G.H.Th., Thessaloniki, Greece

\begin{abstract}
Septic arthritis due to an infection elsewhere in the body is a dangerous disease manifestation. The elderly and particularly those with comorbidities such as diabetes mellitus, arterial hypertension; haemodialysed and the immunocompromised are more susceptible. Infections of the prostate can spread via the blood to cause sepsis and/ or affect joints. Although prostatitis can be diagnosed based on common symptoms and tests, chronic infections and abscesses can be silent and asymptomatic, which allows them to spread. We present a case of a silent prostatic abscess which spread to the hip joint causing septic arthritis and joint destruction. This was further complicated by patient incompliance and refusal for an early surgical debridement. As a result, the condition was aggravated and the joint was lost. The important point made is that clinical presentation of septic arthritis is not always alarming. Moreover, care should be taken to recognise the origin of infection, as it can also be occult. Prompt diagnosis and early treatment are vital, as joint destruction is rapid. Additionally, treatment is complex, dangerous and expensive, with high doses of strong antibiotics, surgeries and variable results. Relapses are common in advanced cases and the hazard for progression to sepsis cannot be neglected.
\end{abstract}

Keywords: Diabetes mellitus, Hip arthroplasty, Hip joint, Prostate abscess, Septic arthritis

\section{Introduction}

In this case report we present an incidence of septic arthritis of the hip due to a prostate abscess. Although rare, prostatic abscesses can be seen after the age of 60 , more often in patients at increased risk. This includes diabetics, immunocompromised, patients on haemodialysis and patients undergoing frequent urinary procedures such as urethral instrumentation or bladder catheterization ${ }^{1-8}$. The commonest identified pathogen is Staphylococcus aureus, while a haematogenous spreading of the infection can result in complications such as sepsis, septic arthritis and osteonecrosis of involved joints ${ }^{1-3,5-8}$.

The significance of this case is the asymptomatic presentation of the prostatic infection (afebrile, without dysuria or frequency), which allowed its haematogenous spreading and the subsequent destruction of the hip joint ${ }^{3,5}$. Additionally, the report demonstrates the difficulties regarding the treatment of progressive septic arthritis and stresses the importance of the patients' compliance with the therapeutic strategy ${ }^{1,3,5,8}$.

\section{Case Report}

A 63 year old male patient presented in the outpatient department of our hospital in December of 2017 due to pain on the right hip. His symptoms had started abruptly 2 months ago with pain initially centred on the right gluteus. This was gradually increasing in intensity and started to radiate to the right hip, until it was finally settled there causing significant restriction of movement. The patient reported no trauma or operations in the affected area and

The authors have no conflict of interest.

Corresponding author: Themistoklis Vampertzis, Department of Orthopedics, Unit for Sport Medicine, Agios Pavlos G.H., Ethnikis Antistaseos 161, 55134, Thessaloniki, Greece

E-mail: themisvamper@yahoo.com

Edited by: Christos Yiannakopoulos

Accepted 30 March 2020 


\begin{tabular}{|c|c|c|}
\hline Test & Result & Reference ranges \\
\hline Haematocrit & $34.6 \%$ & $42-52 \%$ \\
\hline Haemoglobin & $10.8 \mathrm{~g} / \mathrm{dl}$ & $14.0-17.4 \mathrm{~g} / \mathrm{dl}$ \\
\hline Platelets & $435 \times 10^{3}$ & $140-390$ \\
\hline ESR & $105 / h$ & $0-20 / h$ \\
\hline CRP & $10.4 \mathrm{mg} / \mathrm{dl}$ & $<0.5 \mathrm{mg} / \mathrm{dl}$ \\
\hline Glucose & $383 \mathrm{mg} / \mathrm{dl}$ & $70-109 \mathrm{mg} / \mathrm{dl}$ \\
\hline Calcium & $9.3 \mathrm{mg} / \mathrm{dl}$ & $8.4-10.2 \mathrm{mg} / \mathrm{dl}$ \\
\hline ALP & $123 \mathrm{IU} / \mathrm{I}$ & $40-150 \mathrm{IU} / \mathrm{I}$ \\
\hline Leucocytes & $7.99 \times 10^{3}$ & $4.2-11.0$ \\
\hline PSA & $4.990 \mathrm{ng} / \mathrm{ml}$ & $0.000-4.000 \mathrm{ng} / \mathrm{ml}$ \\
\hline
\end{tabular}

Table 1. Blood biochemistry results of clinical relevance upon admission; pathologic results are indicated in bold letters.

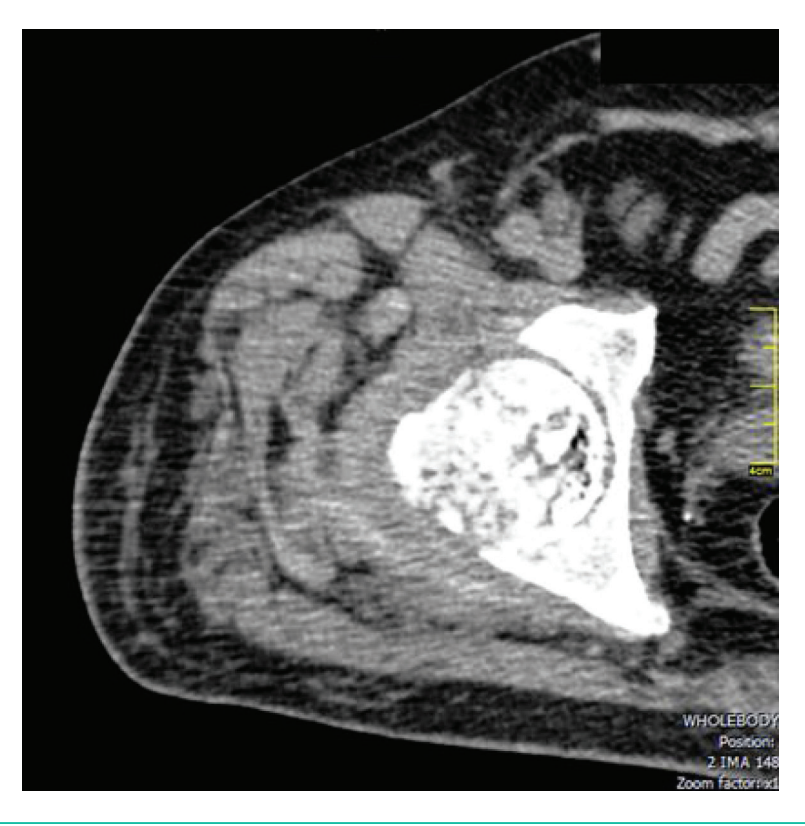

Figure 2. CT of the right hip demonstrating the deterioration of femoral head and the acetabulum.

had been afebrile since the onset of symptoms.

Patient was a chronic type II diabetic, suffered from arterial hypertension and had a Cerebrovascular Accident in 2005. He was a social drinker and had quit smoking 15 years ago. He reported no known allergies and had a clear family history.

Upon clinical examination the right extremity was held in a flexed position for pain alleviation. Great discomfort was elicited by only minor pressure on the right hip. Active and passive movements were limited, particularly extension and

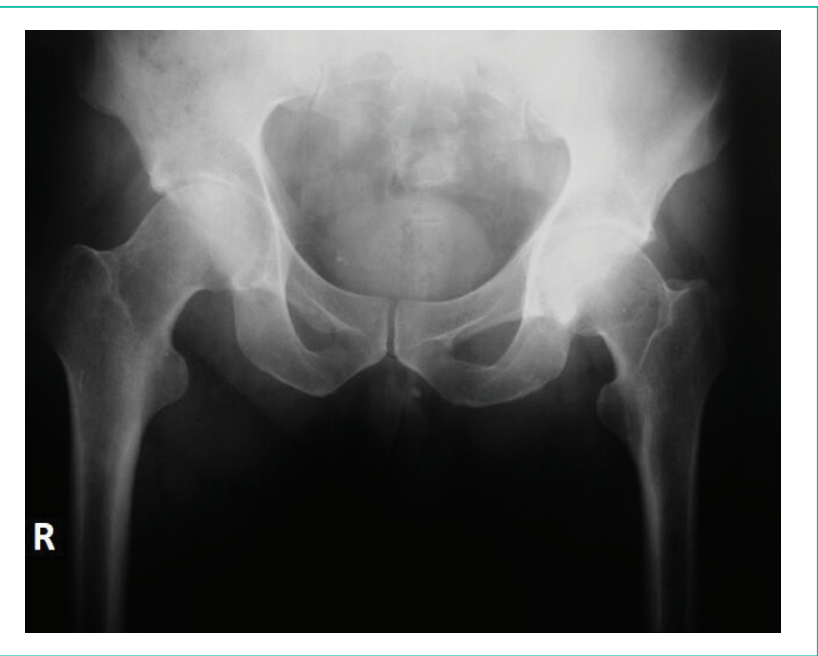

Figure 1. X-ray of the hips upon admission, showing bilateral osteoarthritic changes.

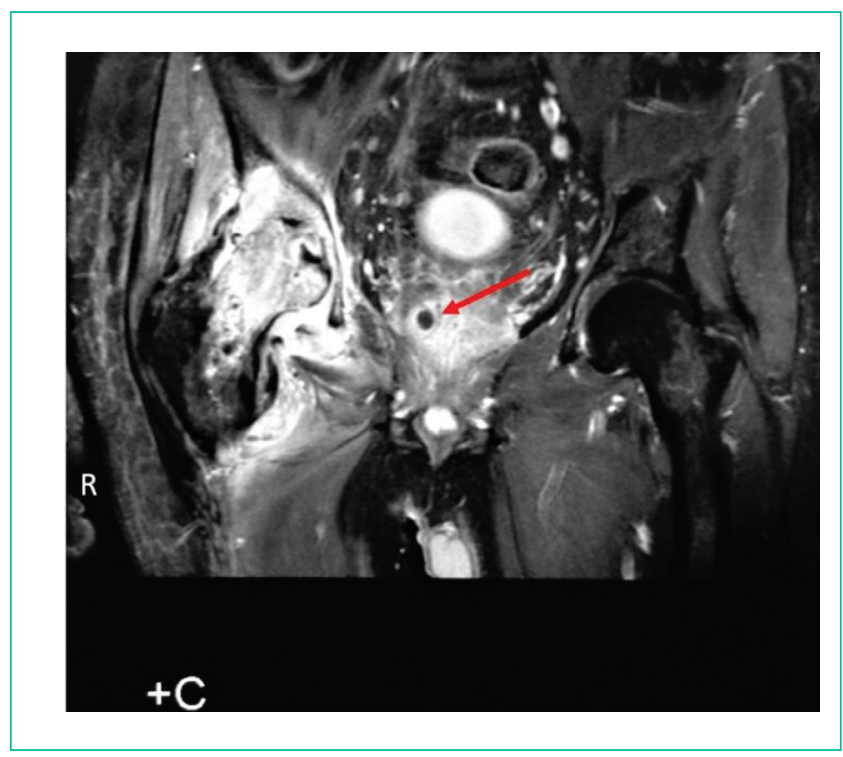

Figure 3. Coronal view of the right hip and the prostate abscess (arrow) on MRI.

internal rotation. Thomas sign was positive, but the patient was unable to undergo the Trendelenburg test. Blood tests and $\mathrm{X}$-rays were ordered (Table 1 ).

On X-ray significant osteoarthritic changes were identified bilaterally on the hips, more prominent on the right (Figure 1). At this point the patient was admitted to the hospital for normalization of his blood results and further investigation of the underlying condition.

A CT scan of the right hip revealed multiple erosivecorrosive, lytic deteriorations of the femoral head and the 


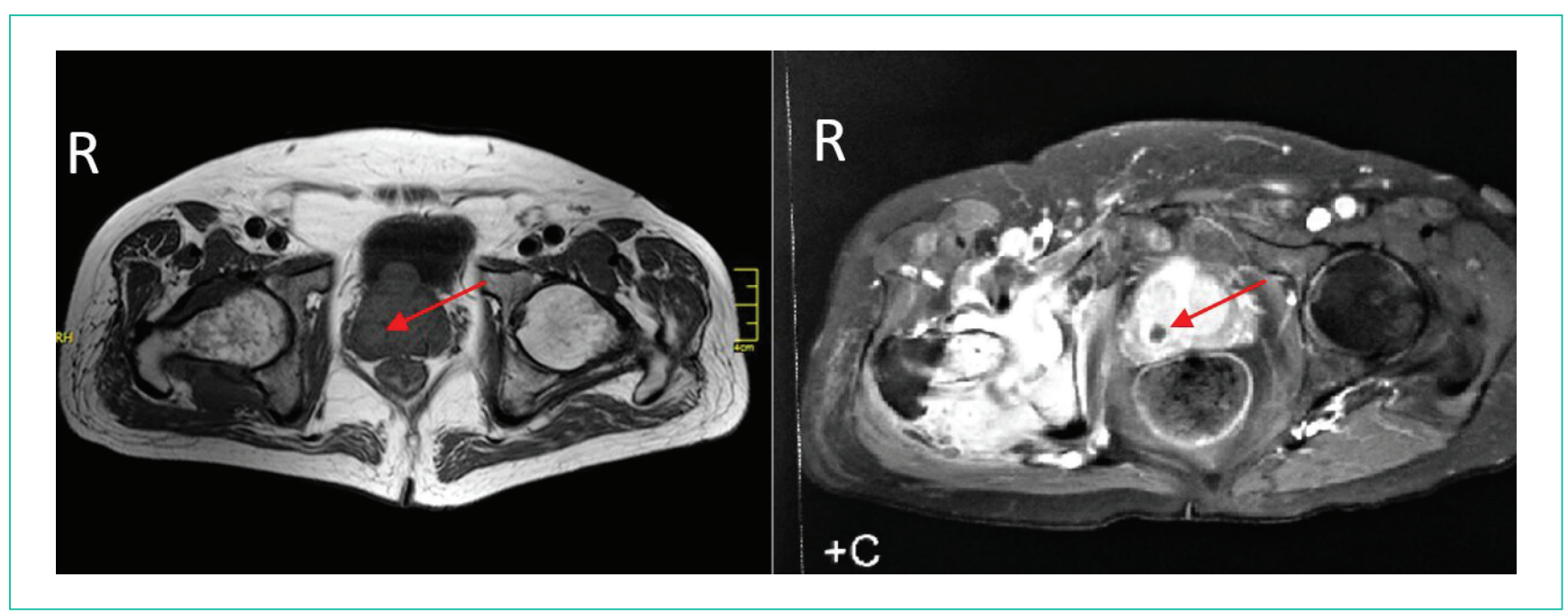

Figure 4. Axial view of the right hip and the prostate abscess (arrow) on MRI, without (left) and with (right) the use of contrast material.

acetabulum, intra-articular space narrowing, folliculitis and atrophy of the right gluteal muscle (Figure 2). A bone scan confirmed the CT findings, also demonstrating flattening of the femoral head and soft tissue infiltration around the femoral neck. An MRI was ordered on which crescent degeneration of the femoral head was evident. There were pathologic intra-articular follicular collections and affection of the acetabulum and femoral head and neck to the right, with infiltration of the adductor muscles. As an incidental finding an abscess in the prostate gland was identified (Figures 3, 4).

The differential diagnosis at this point included rapidly destructive arthritis, septic arthritis, Avascular Necrosis of the femoral head, neoplasia, tuberculous arthritis and Charcot's joint ${ }^{8}$. Due to the presence of the prostatic abscess and the suspicious laboratory results (Table 1), septic arthritis had to be investigated.

A C-arm guided paracentesis of the right hip joint was ordered to collect intra-articular fluid for culture. Simultaneously Urologists were consulted. Culture results revealed a Methicillin Susceptible Staphylococcus aureus (MSSA) infection of the joint, a commonly identified pathogen in similar cases ${ }^{1-3,5-8}$. A culture of the prostate confirmed that the infection had spread from the prostatic abscess. The patient was informed that the best approach was a surgical debridement but insisted on being treated conservatively for the infection and return home despite understanding the hazards (loss of the joint, severe sepsis and death) ${ }^{1,3,8}$. Therefore, based on antibiogram, intravascular Teicoplanin $1 \times 2$ was administered for two weeks ${ }^{3,6,8}$. Following normalization of inflammatory markers the patient was discharged on Ciprofloxacin 500 mg $1 \times 2$ for 3 months. He was instructed not to load the extremity (use crutches) and to visit the outpatient department every 15 days.

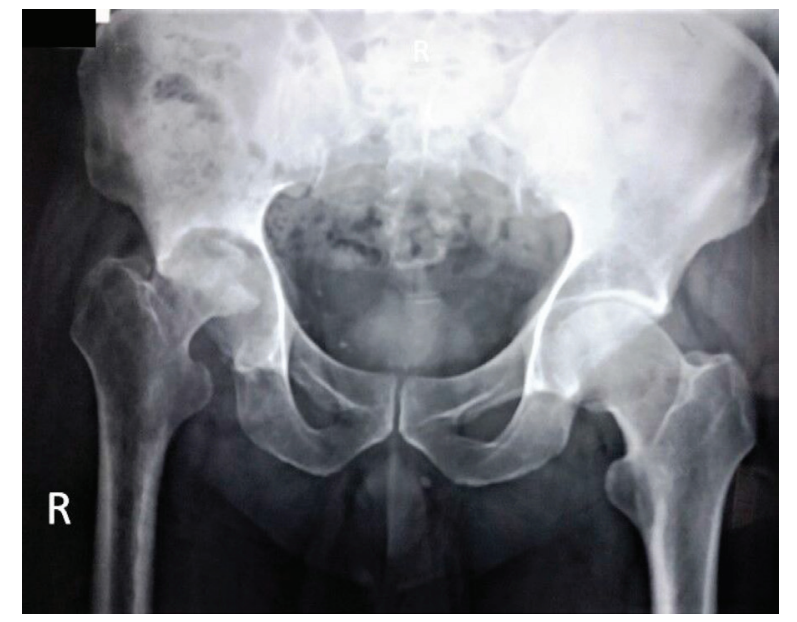

Figure 5. X-ray of the hips showing the deterioration of the necrosis on the right.

Due to incompliance of the patient with follow-ups and medication he presented 3 months after discharge with worsening of the osseous deterioration. An X-ray and a CT showed progression of the necrosis (Figures 5,6 ); the extremity was shortened by $4 \mathrm{~cm}$ and inflammatory markers were high (ESR 55/h, CRP $3.9 \mathrm{mg} / \mathrm{dl}$ ). The new therapeutic strategy was a hip arthroplasty in two stages, with which the patient agreed ${ }^{1,9}$. The acetabulum was scraped, the inflamed tissue was removed and a cement Spacer loaded with calcium sulphate dehydrate and gentamycin was placed (Figure 7$)^{1,9}$. After recovery, 


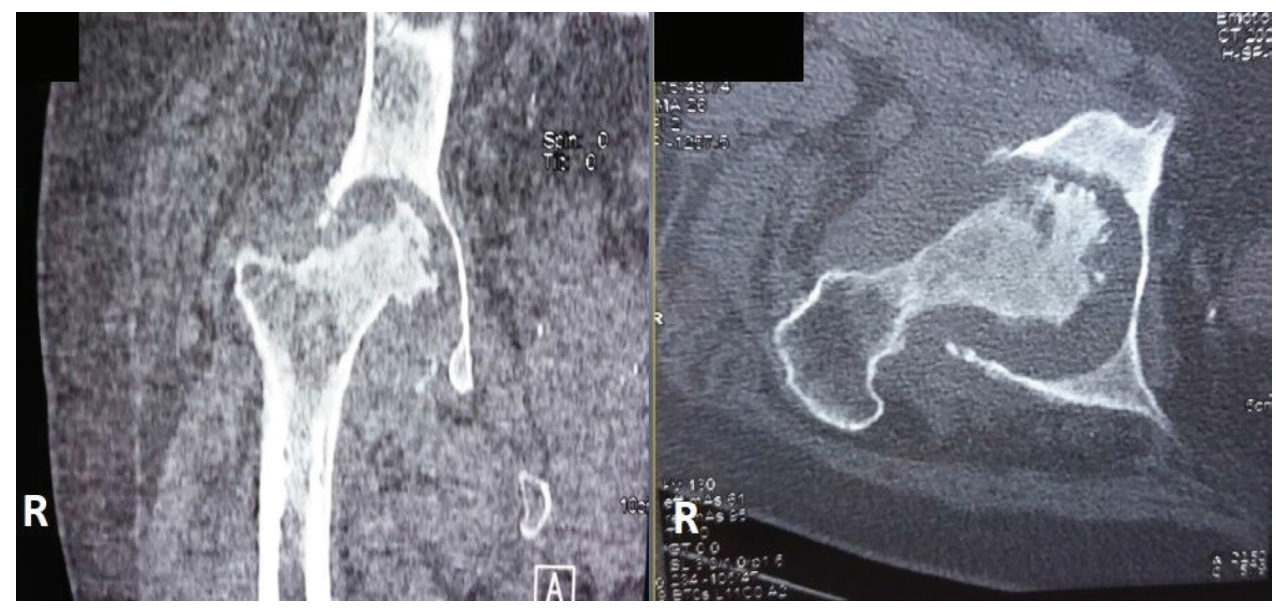

Figure 6. CT of the right hip showing the progression of the osteonecrosis on the coronal (left) and axial (right) planes.

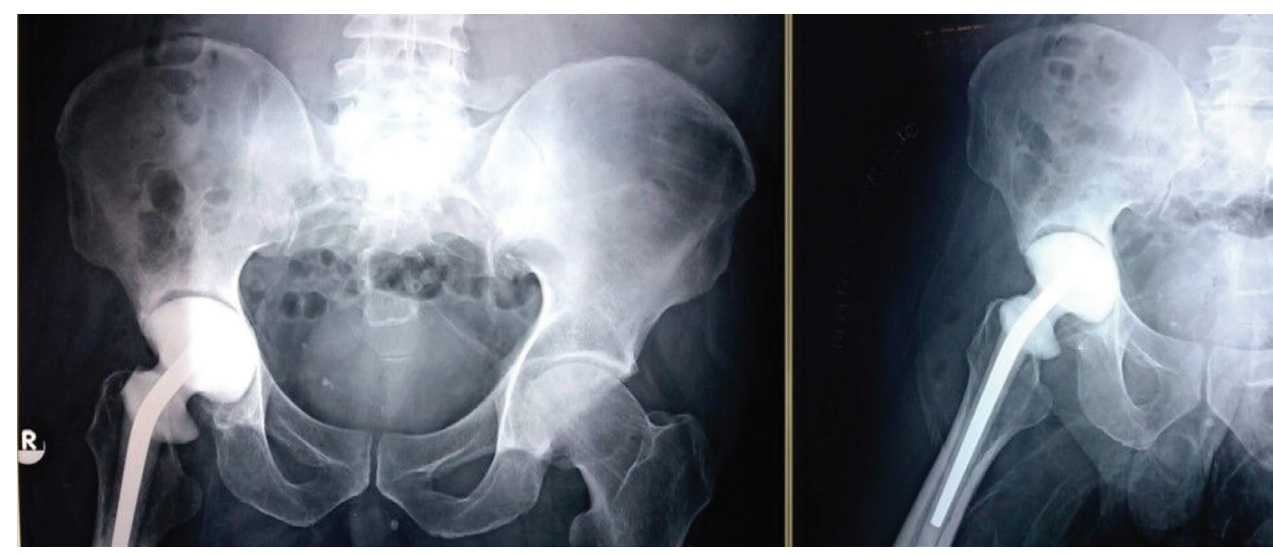

Figure 7. Post-operative X-rays after the placement of a Spacer.

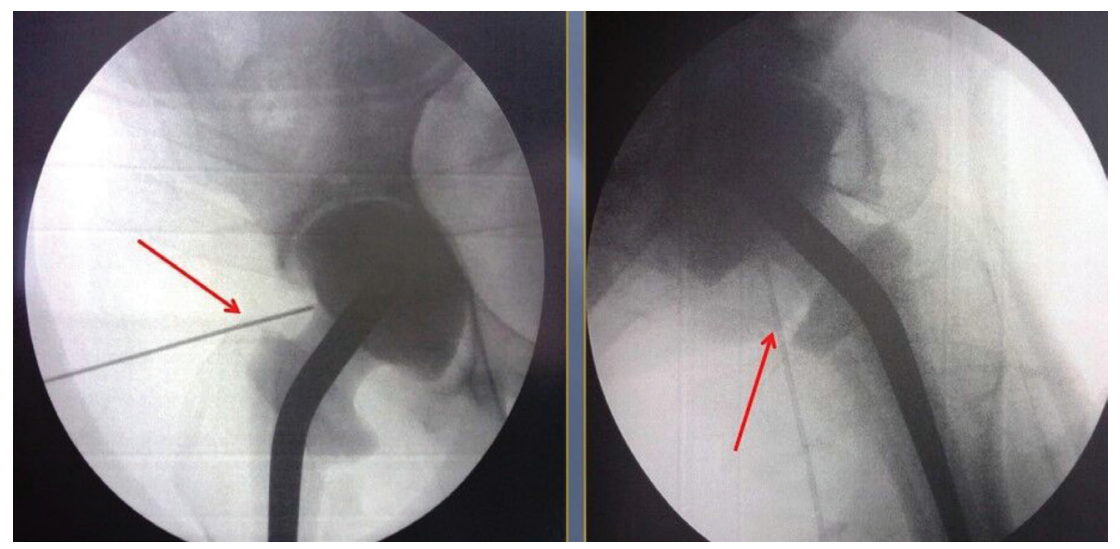

Figure 8. C-arm guided paracentesis of the right hip, red arrow indicating the paracentesis needle. 


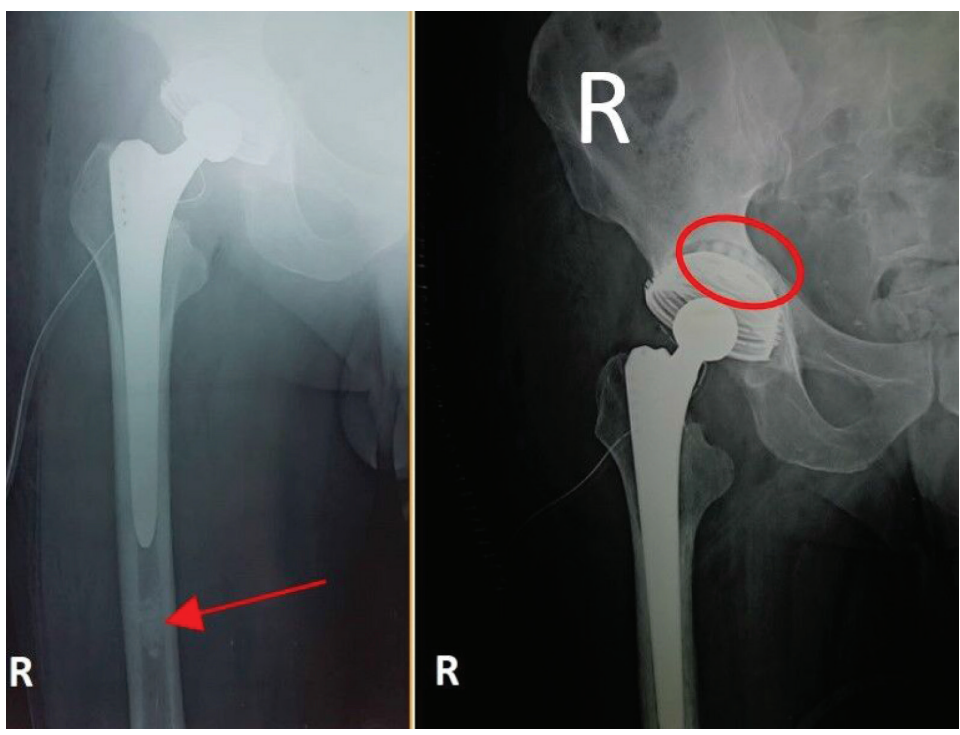

Figure 9. Post-operative X-rays after the total hip arthroplasty with placement of antibiotic loaded beads on the femur (left, arrow) and on the acetabulum (right, circle).

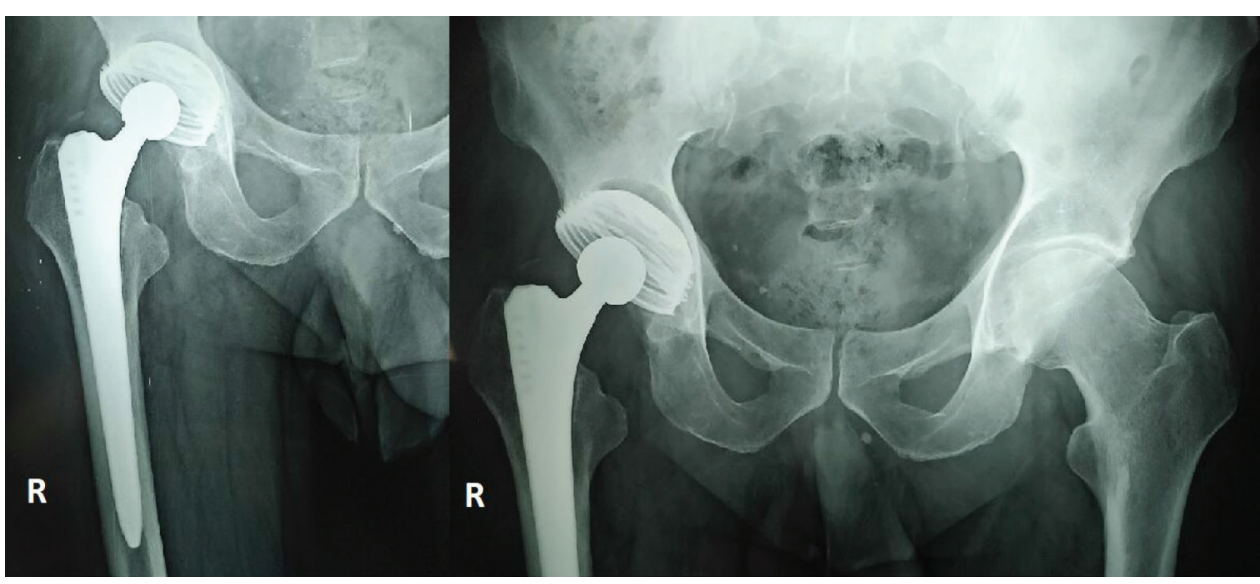

Figure $10 . \mathrm{X}$-rays in the $9^{\text {th }}$ post-operative month.

patient was discharged on Ciprofloxacin $(500 \mathrm{mg}$ 1x2) for 2 months, Clindamycin (300 mg $1 \times 4$ ) for 1 month and instructions for partial weight bearing using crutches. Three months later a one-month window off antibiotics was allowed to obtain a new intra-articular fluid sample for culture (Figure 8) and to perform blood tests. With negative culture results, no inflammatory markers, normal PSA and no signs of infection a total hip arthroplasty (uncemented) was done with placement of calcium sulphate dehydrate, hydrogenated triglyceride and gentamycin sulphate beads (Figure 9) ${ }^{9}$. A week later the patient was discharged on antibiotics for one more week. Regular laboratory controls and radiographic investigations were done every 10 days during the 1 st month and once every subsequent month. In the 9th post-operative month the patient was asymptomatic and able to walk unassisted with full loading of the extremity (Figure 10). 


\section{Discussion}

Septic arthritis of the hip joint does not necessarily present with an alarming clinical picture; in many cases the only complaint is pain and restriction of movement. Similarly, prostatic infections which are frequent in elderly susceptible groups also have variable presentation ${ }^{1-8}$. Therefore, diagnosis can be delayed allowing for the haematogenous spreading of the infection, the development of septic arthritis and the subsequent destruction of the affected joint ${ }^{2-8}$. Reported in literature are among others, cases of septic arthritis of the hip, the knee, the trapezio-metacarpal and sternoclavicular joints, as well as the joints in the spine ${ }^{1,4,6-8,10}$. This report identifies a clinically silent prostatic abscess as the cause of the infection and the complete destruction of the hip joint. Treatment itself is difficult, longterm and with high socio-economic impact; while similarly to all bone inflammations relapses and complications cannot be excluded $^{1-3,5,8,9}$.

\section{References}

1. Anagnostakos K, Duchow L, Koch K. Two-syage protocol and spacer implantation in the treatment of destructive septic arthritis of the hip joint. Arch Orthop Trauma Surg 2016;136(7):899-906.

2. Domingue GJ Sr, Hellstrom WJ. Prostatitis. Clin Microbiol Rev 1998; 11(4):604-13.
3. Jang K, Lee DH, Lee SH, Chung BH. Treatment of prostatic abscess: case collection and comparison of treatment methods. Korean J Urol 2012;53(12):860-4.

4. Lepetit C, Le Gal S, Michon J, Collon S, Tillou X. Pseudomonas aeruginosa septic trapezo-metacarpal arthritis after prostate laser vaporization. Infection 2015;43(4):503-5.

5. Souza Miyahara Hd, Helito CP, Oliva GB, Aita PC, Croci AT, Vicente JR. Clinical and epidemiological characteristics of septic arthritis of the hip, 2006 to 2012, a seven-year review. Clinics 2014; 69(7):464-8.

6. Shioya N, Ishibe Y, Kan S, Masuda T, Matsumoto N, Takahashi G, Makabe H, Yamada Y, Endo S. Sternoclavicular joint septic arthritis following paraspinal muscle abscess and septic lumbar spondylodiscitis with epidural abscess in a patient with diabetes: a case report. BMC Emerg Med 2012;12:7.

7. Okano T, Enokida M, Otsuki R, Hagino H, Teshima R. Recent trends in adult-onset septic arthritis of the knee and hip: retrospective analysis of patients treated during the past 50 years. $J$ Infect Chemother 2011;17(5):666-70.

8. Munoz-Egea MC, Blanco A, Fernandez-Roblas R, Gadea I, Garcia-Canete J, Sandoval E, Valdazo M, Esteban J. Clinical and microbiological characteristics of patients with septic arthritis: A hospital-based study. J Orthop 2014;1 1(2):87-90.

9. Beuerlein MJ, McKee MD. Calcium sulphates: what is the evidence? J Orthop Trauma 2010;24:46-51.

10. Matsumoto M, Shigemura K, Yamamichi F, Nakano Y, Miyake H, Tanaka K, Arakawa S, Fujisawa M. Hinyokika Kiyo. A case of prostate abscess with sepsis, infectious endocarditis and pyogenic spondylitis 2012;58(10):565-8 